\title{
无机及分析化学实验 “三维” 教学模式的设计与实践
}

\author{
周雅情, 梅芯, 吴文海* \\ 汉江师范学院化学与环境工程学院, 湖北十堰 442000
}

\begin{abstract}
摘要: 无机及分析化学实验是汉江师范学院生物科学专业在大一开设的首门必修实验课程, 其教学质量对后续专业 课程的学习、学生学术研究能力和创新性思维的培养等具有重要意义。然而, 汉江师范学院无机及分析化学实验教 学过程中存在理论课程与实验课程教学进度不匹配、学生课前预习不充分以及课程期末考核方式单一等问题。笔者 通过课件推送、问题收集、能力提升的 “三维” 教学模式, 突出学生的主体地位, 将实验课程的考核机制落实到课 程学习的各个环节, 旨在提高学生的学习动力和激发学生的求知欲。该 “三维” 教学模式的实施对提高无机及分析 化学实验课程的教学质量具有重要意义。
\end{abstract}

关键词: 无机及分析化学实验; 生物科学; 三维; 考核机制; 学习动力; 教学质量 中图分类号: G64; O6

\section{Design and Practice of the "Three-Dimensional" Teaching Mode for Inorganic and Analytical Chemistry Experiments}

\author{
Yaqing Zhou, Rui Mei, Wenhai Wu * \\ College of Chemical and Environmental Engineering, Hanjiang Normal University, Shiyan 442000, Hubei Province, China.
}

\begin{abstract}
Inorganic and analytical chemistry experiment is the first offering compulsory laboratory course of biological science specialty in Hanjiang Normal University, whose teaching calibre is of great significance to the followup specialty course study, and the cultivation of research capabilities and innovative thinking of the undergraduates. However, such course has some issues including unmatched schedule between theory and practice, unpreparedness for class and monotonous final examining mode. The "three-dimensional" teaching mode comprised of courseware transmission, question collection and ability enhancement were employed to improve the learning initiative and stimulate curiosity by highlighting the principal position of students and implementing the assessment mechanism to each link of the course learning. The development of the "three-dimensional" teaching mode is applicable in facilitating the teaching quality of inorganic and analytical chemistry experiment course for Hanjiang Normal University.
\end{abstract}

Key Words: Inorganic and analytical chemistry experiment; Biological sciences; Three-dimensional; Assessment mechanism; Learning motivation; Teaching quality.

实验教学在人才培养中发挥着不可替代的基础作用, 是培养学生动手能力、科研素养、创新创 业能力和可持续发展能力的重要依托 ${ }^{[1]}$ 。在过去近 25 年中, 我国的化学实验教学改革取得了巨大的 进步, 但地方高校化学实验教学现状仍然不太乐观, 尤其是新进专升本的高校, 存在着教学方法传 统、教学管理落后和考核方式单一等问题。这一类高校亟需进行教学改革, 以适应本科教学和发展

收稿: 2021-06-06; 录用: 2021-08-11; 网络发表: 2021-09-07

“通讯作者, Email: wuwenhai34@126.com

基金资助：博士科研启动金(506030030214) 
的需要。本文以2016年专升本的地方高校汉江师范学院为研究对象, 对无机及分析化学实验课程进 行教学改革, 旨在与同行交流，以便不断地提高教学质量，为同类高校提供借鉴。

无机及分析化学实验是汉江师范学院非化学专业生物科学的学科基础课, 旨在使学生掌握基本 的操作技能和实验技术, 培养学生观察问题、分析问题、思考解决问题和独立操作实验的能力, 养 成严谨的学术研究素养, 树立开拓的创新意识, 为后续生物科学专业的各类专业课程学习和实验技 术奠定坚实的基础。然而, 汉江师范学院无机及分析化学实验教学过程中存在诸多问题, 如: 理论 课程与实验课程的教学进度不匹配、学生课前预习不充分、课程期末考核方式单一、学习积极性差、 创新性不足等问题, 不能满足新时代对创新性人才培养的要求。因此, 笔者针对以上问题, 设计了 “三维” 教学模式, 即 “课件推送 $\rightarrow$ 问题收集 $\rightarrow$ 能力提升”。“课件推送” 从学生角度出发, 课前 线上推送课件, 要求学生提前预习实验内容, 完成思考题的解答; “问题收集” 从教师角度出发, 收集学生预习过程中提出的问题, 针对问题着重讲解, 对症下药, 提高课堂效率; “能力提升” 则 是综合课前预习、课中讲解, 从而总结反省, 找出不足, 针对学生与教师的不足之处进行改进, 完 成量变到质变的转换。教学过程中以学生为主体, 将实验教学的考核机制落实到课程学习的各个环 节, 旨在提高学生的学习动力, 提高汉江师范学院无机及分析化学实验课程的教学质量。这种多层 次、多对象的 “三维” 教学模式的实施, 不仅能够加深学生对理论知识的理解, 培养学生独立思考、 操作、正确归纳和综合处理数据的能力, 还能帮助学生形成良好的科学思维习惯, 从而逐步掌握科 学研究的方法 ${ }^{[2]}$, 为后续专业课和实验课的学习夯实基础。同时, 还能帮助教师革新教学方法, 提高 教学水平, 以学促教, 教学相长。本篇文章着重介绍了汉江师范学院无机与分析化学实验课程的教 学现状、存在问题及 “三维” 教学模式的设计与实施。

\section{1 无机及分析化学实验课程的教学现状}

\section{1 理论课程与实验课程教学进度的匹配性}

目前, 汉江师范学院生物科学专业无机及分析化学实验课程一学期开设 16 个实验, 45 个学时, 其中无机实验 5 个, 包括: 1 个准备实验, 2 个基础实验, 2 个制备实验; 分析实验 11 个, 包括: 5 个标 定实验, 6 个测定实验。无机及分析化学理论课程是先学习无机化学知识, 再学习分析化学知识, 因 此, 5 个无机化学实验能够与理论课讲授的进度一致, 大一新生可以在学习理论知识后, 通过实验的 操作进一步加深对理论知识的理解 ${ }^{[3]}$ 。分析部分的实验主要以验证性实验为主, 涉及滴定原理、正 确判断终点、滴定操作和指示剂选择等知识点, 但实验课超前于理论课, 没有理论知识支撑, 使得 学生在实验操作中出现 “照方抓药” 的现象, 同时大一新生在实验过程中不能完全理解实验的过程 和现象, 更不知道如何解决实验过程中出现的问题 $\left.{ }^{4}\right]$ 。而学习到理论知识时, 对应的实验早已完成, 做实验时未能理解透彻理论知识点, 使得学生未能将实验与理论知识有效结合, 导致没有达到有效 的教学, 不利于学生综合素质的培养和创新能力的提高 ${ }^{[5]}$ 。

\section{2 传统的教学方法}

目前, 汉江师范学院的实验教学方法是 “课前预习(课前检查预习报告) $\rightarrow$ 教师为中心的讲授 $\rightarrow$ 学生完成实验操作 $\rightarrow$ 学生完成实验报告 $\rightarrow$ 根据实验报告评定平时成绩 $\rightarrow$ 期末理论知识考查”。主要 反映出以下几个问题: (1) 实验课前, 学生预习是实验课的关键, 但学生课前实验预习仅停留在文字 层面, 未能直观地感知实验过程和仪器的使用, 课堂上仅依赖教师的课堂演示, 机械地完成实验, 一段时间已忘记, 不利于培养学生的创新性思维和科学探究态度; (2) 实验课中, 教师讲解和演示的 时间过长, 占用了学生实验操作的时间, 由于实验课学时和实验条件的限制, 学生在短时间内机械 与盲目地完成实验, 在实验中存在的疑惑和问题缺乏有效的与教师和同学讨论的渠道, 一旦实验失 败, 学生没有过多的时间再次进行探究, 不利于培养学生观察问题、分析问题、思考解决问题和独 立实验操作的能力, 这样的教学方式容易使学生失去学习兴趣, 且极大地限制了学生的思维发展 ${ }^{[6,7]}$, 同时不利于学生对基本仪器操作的掌握及加强对学生进行实验规范操作能力的培养; (3) 实验课 
后, 教师很难根据学生上交的实验报告正确评价学生的实验操作能力, 使得教师不能有效指导学生 基本操作和实验技术; (4) 课程考核, 根据实验报告的书写评定学生的平时成绩, 但实验报告仅反映 了学生对实验的理解和书写的认真, 不能反映出学生操作实验的真实情况, 不乏有学生为了高分的 成绩篡改数据、抄袭等。期末理论知识考查仅测试了学生的理论知识, 未能考查学生独立思考、准 备和实践操作的能力, 导致实验教师不能全面评估学生的实验能力。

\section{3 学生实验基础知识薄弱}

在当前高等教育进入普及化的新阶段, 以汉江师范学院首届 2020 级生物科学专业51名本科生为 研究对象, 在进校学习两周后, 采用问卷网匿名方式对 “大学生实验学习情况” 进行调查, 回收问 卷 51 份, 问卷回收率为 $100 \%$, 除去重复提交的问卷 2 份, 有效问卷回收率为 $96.1 \%$, 结果如图 1 所示。 从问卷调查结果显示: (1) $28.6 \%$ 的学生从未做过实验, $71.4 \%$ 的学生偶尔做实验, 更有 $8.2 \%$ 的学生从 未接触过实验仪器, 可以得出学生的实验基础知识和实验基本操作能力薄弱; (2) 课前预习, 仅有 $28.6 \%$ 的学生经过预习可以完成实验, 由此可以得出学生课前预习仅是形式化, 学生的真实情况未能 及时反馈给任课教师, 致使有些知识学生未能掌握, 任课教师只能花费大量时间讲解理论知识, 使 得学生在有限的实验课上只能按部就班地完成实验, 不利于大学生学术研究和创新意识的培养; (3) 从4、5、6三个问题的调查结果显示: 学生思考问题能力、学习能力和解决问题的能力等存在不 一致性, 在课堂有限时间内无法满足不同水平学生学习的需要。

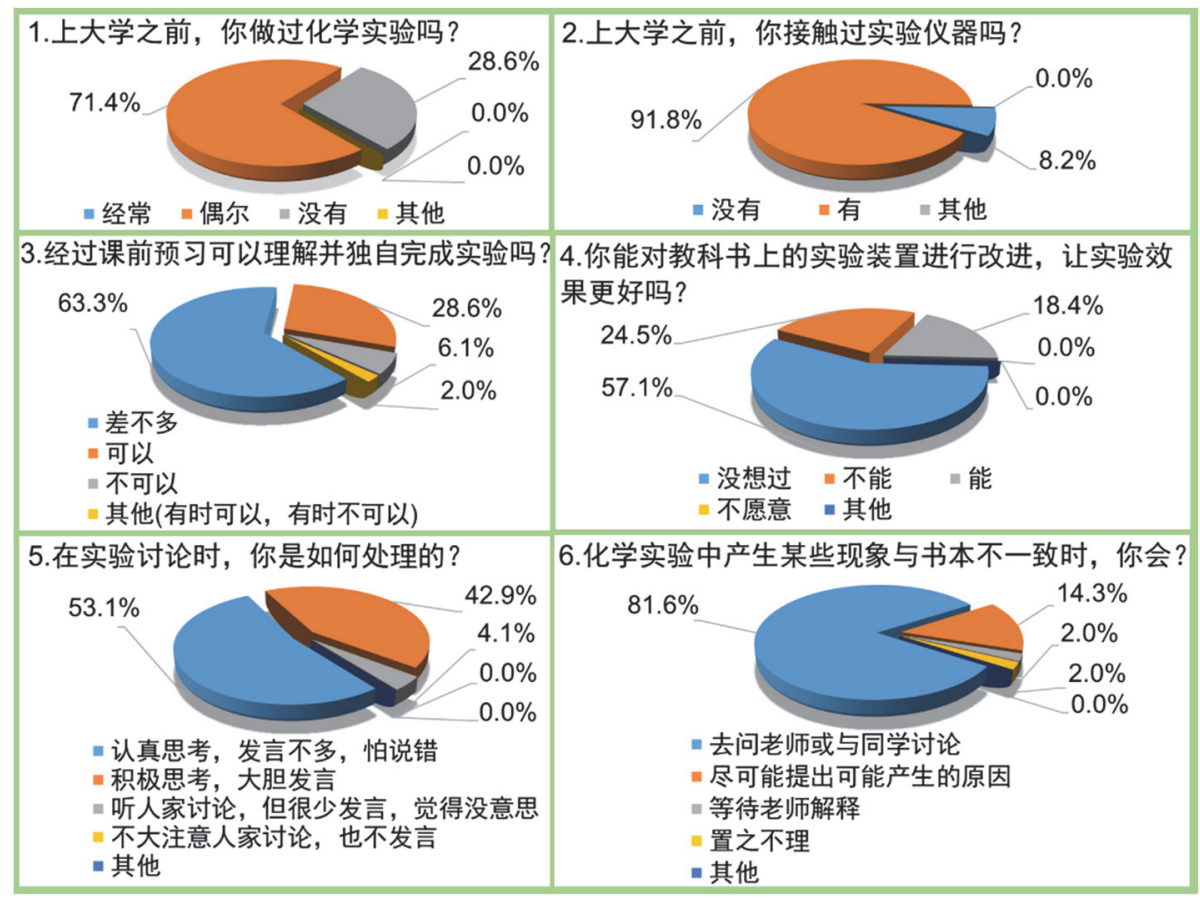

图1 大学生实验学习情况结果一问卷网调查

电子版为彩图

\section{2 无机及分析化学实验 “三维” 教学模式的设计 \\ 2.1 “三维” 教学模式的设计理念}

汉江师范学院大一新生均是 00 后, 传统的教学方法适用于 80 年代和 90 年代的学习者, 但很难调 动 00 后学生的学习动力和积极性。相比于 80 年代和 90 年代的学生, 00 后更早接触了信息技术, 他们 的思维更加活跃、有思想、有目标、有激情, 但以自我为中心, 具有自律意识、自主意识、合作意 识薄弱等问题。问卷网调查结果显示了以下问题：学生实验基础知识薄弱、基本操作能力薄弱、课 
前预习形式化、学生实验能力参差不齐。在汉江师范学院 00 后学生中, 缺乏自主意识和学习动力弱 者占大部分, 而学习能力强、充满求知欲和好奇心者仅占小部分。因此, 如何将学习动力弱的学生 培养成学习动力强的学生, 是提高实验课程教学质量的根本, 但也存在着挑战, 主要是学生缺乏 “直 接需求” 的学习动力, 本课程的教学设计只能针对他们的 “间接需求” , 即增加课程的考核强度和 水平 ${ }^{[8]}$ 。结合汉江师范学院的实际, 借鉴国内高校基础实验课程先进的教学设计理念 ${ }^{[9-12]}$, 在教学过 程中运用互联网技术一问卷网、雨课堂等现代教学手段, 设计了 “三维” 教学模式, 即 “课件推 送 $\rightarrow$ 问题收集 $\rightarrow$ 能力提升”。第一个维度 “课件推送”, 从学生角度出发, 旨在寻找问题根源; 第 二个维度 “问题收集”, 从教师角度出发, 旨在寻找问题解决方法, 更进一层, 以点扩面; 第三个 维度 “能力提升”, 综合学生与教师的两个角度, 面面俱到, 层层递进, 形成更高的维度。转变传 统的教师讲学生听的教学模式, 让学生成为课堂的主体, 积极主动研究课程内容, 提出自己的想 法, 激发学生的兴趣, 将课程考核机制落实到课程学习的各个环节, 旨在提高学生主动学习的动力, 以及提高无机及分析化学实验课程的教学质量。

\section{2 “三维” 教学模式的实施和课程考核机制}

教学设计和课程考核计分如图2所示。具体实施过程分析如下:

(1) 课前学生自主预习实验。课前 1 周, 教师明确课程预习任务: (1) 雨课堂推送教学课件, 包 括: 实验详解(实验目的、原理、内容)及注意事项、操作视频及其他资料, 以及设置的思考题; (2) 问 卷网推送给每位学生, 调查包括: 不懂的实验理论知识点、需要着重讲解的实验理论知识点及需要 演示的实验操作。学生在课前完成预习任务, 课堂随时提问考核。

其中, 课前学生通过雨课堂推送的教学课件, 明确了课堂教学内容, 有针对性地预习。雨课堂 设置思考题并设置分数, 学生须课前完成答题(学生可以文字描述, 也可以在雨课堂用语音回答问 题), 教师及时批改并记录分数。有效防止了部分学生的实验预习仅停留在文字层面, 懒于查找资料 和独立思考, 课堂上不能很好地接受教学内容, 导致实验操作出现较多的问题和机械式的参照实验 步骤做实验; 有效检验了学生的实验预习情况, 同时设置语音回答思考题, 提高了学生的学习兴趣。 课堂上针对性的反馈分数, 不仅促进了学生预习实验的能动性, 还培养了学生的学习习惯(善于思考、

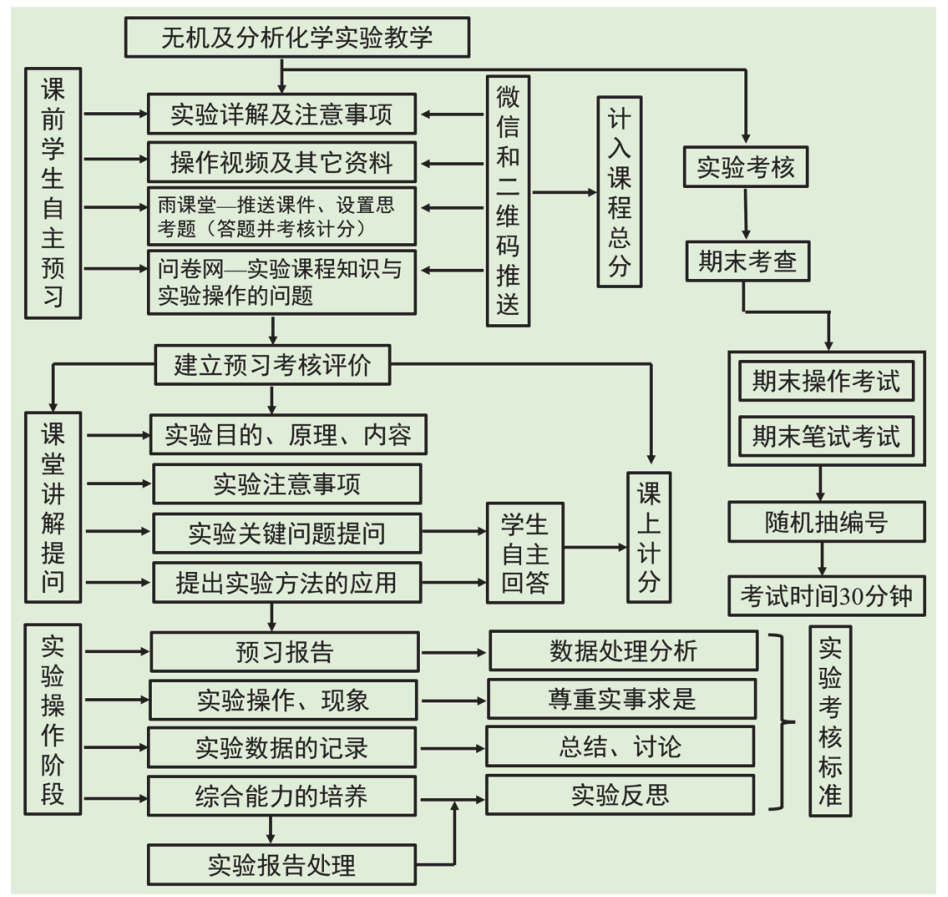

图2 无机及分析化学实验 “三维” 教学模式的设计流程图 
自主学习、独立解决问题等), 同时教师可以在雨课堂上直观了解学生实际的预习情况, 将预习情况 和答题情况纳入平时成绩考核中。操作视频主要针对实验的基本操作演示, 例如 “分析天平的使用、 酸式滴定管的使用”, 可以让学生更直观地了解实验的操作, 并且可以在任何时间反复观看, 同时 弥补了在传统实验课堂教学中学生操作能力参差不齐的不足, 尤其是操作的细节, 有效避免了在课 堂上有限的时间内演示、实验操作细节错误、实验失败情况。问卷网提前推送给每位学生, 做到对 症下药、有的放矢, $80 \%$ 学生都懂的知识点简单提一下, $50 \%-80 \%$ 学生懂的知识点简单讲解, $50 \%$ 以 下学生提出的实验课程知识与实验操作问题, 在课堂上着重讲解和演示, 避免了在有限的课堂上大 篇幅讲解学生已经知晓的知识, 同时满足了不同水平学生学习的需要。

(2) 课堂讲解提问。

课前反馈雨课堂设置思考题的分数, 分数高的学生受到教师的表扬和给予肯定, 同时获得同学 们的掌声, 让学生感受到情感体验一自豪感、成就感和荣誉感, 调动学生的积极性, 激发学生的 学习动力。课堂讲授环节主要突出了学生的主体地位, 在讲授过程中提出问题, 由学生自主回答, 同时引导学生之间相互交流、分享自己的想法, 旨在锻炼学生回答问题的逻辑性、语言表达能力和 互相合作的能力, 努力培养学生成为课堂中的主体认知, 同时, 未准备充分的学生将受到减分的惩 罚。设置问题环节中会设置实验拓展难题, 增设附加分值, 当场加分奖励, 提高学生的积极性和激 发学生的求知欲。问卷网推送收集实验课理论知识和实验操作存在的问题, 如实验 “食醋中总酸度 的测定” , 笔者设置的实验材料是市场上售卖的食醋, 雨课堂设置思考题 “滴定反应式是什么? ”。 有学生在问卷网上提出问题, “食醋中的主要成分是醋酸, 此外还含有少量的其他弱酸如: 乳酸、 柠檬酸、葡萄酸、苹果酸等, 虽然食品中含有多种有机酸, 总酸度测定结果常以样品中含量最多的 那种酸表示, 滴定反应式应是: $\mathrm{CH}_{3} \mathrm{COOH}+\mathrm{NaOH}=\mathrm{CH}_{3} \mathrm{COONa}+\mathrm{H}_{2} \mathrm{O}$, 那市售的柠檬是否可以用 同样的方法测出酸度? ” 还有学生提出 “橘子可以用此方法测出酸度吗? ” 等等。问卷网平台反馈 的信息显示学生对此实验比较感兴趣, 在课堂上针对此问题展开了讨论, 引导学生提出实验方法的 应用, 并准备了少量的柠檬和橘子, 在实验课程完成后, 有兴趣的做了柠檬和橘子中酸度的测定, 整个教学过程中学生的学习兴趣高涨, 提高了课堂的教学质量, 同时纳入平时成绩考核中。

(3) 实验操作阶段。

学生独立准备和操作实验, 细致观察和记录实验现象, 正确处理实验数据和正确分析实验结果, 实验结束后, 教师检查预习报告(实验的记录和数据的处理), 并根据学生在实验过程中的操作, 当场 考核打分, 记录平时成绩。

(4) 实验报告处理。

学生完成实验课程的常规内容: 数据处理分析、总结、讨论。要求每位学生完成实验后, 针对 在实验中出现的问题及成功完成实验的关键操作给予反思, 并写在实验报告本上, 旨在让学生加深 对本实验的学习, 学会分析实验误差的原因, 考核计分如图3所示。

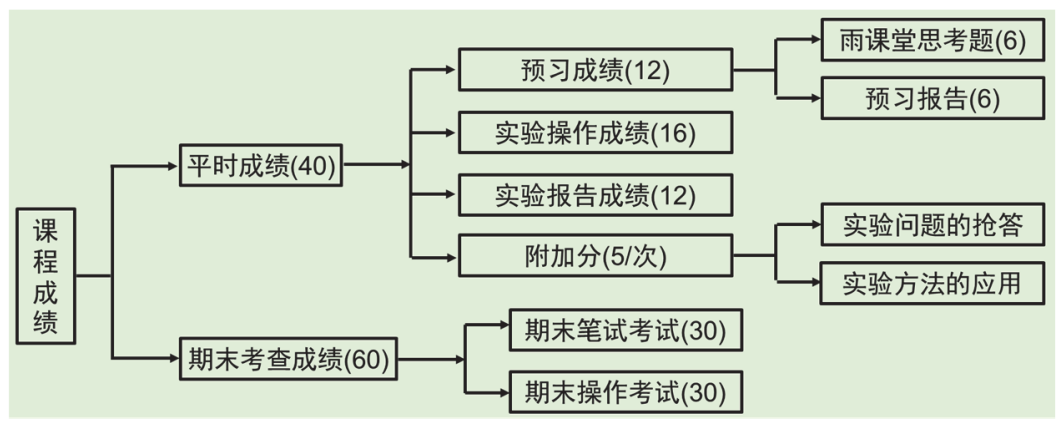

图3 无机及分析化学实验课程考核标准 
(5) 课程期末考核。

无机及分析化学实验课程考核标准包括平时成绩和期末考查成绩两部分。整个课程考核均制 定了详细的计分标准(如图3)。期末考查: (1) 期末笔试成绩一一理论知识考查: 主要考查无机及分析 化学实验课程的一般知识、基本概念、实验原理、实验数据处理以及思考题; (2) 期末操作成绩一一实 验操作考查: 8 个实验随机抽取其中 4 个实验进行考查, 涉及仪器识别、实验的操作、数据处理、操 作注意事项等。所有学生分成若干组, 每组学生进场考试时间不同, 提前 30 分钟候考, 每组学生随 机抽取编号, 编号对应不同的理论知识和实验操作, 避免了互相抄袭的行为, 考试时间 30 分钟, 在 规定的时间内完成理论和操作考试, 时间到停止考试, 结束后学生离场, 并不能与候考的学生交流, 所有的考核环节均设置计分标准, 避免了考核片面和不公平计分的弊端。课程考核不是目的, 将课 程考核落实到课程学习的各个环节, 意在提高学生主动学习的动力和培养学生学术研究的能力。

\section{3 无机及分析化学实验 “三维” 教学模式的教学效果}

“三维” 教学模式取得的教学成效: (1) 经过2次课程的开展, 生物科学专业的全体学生已养成 了课前预习的习惯, 课前能充分地预习雨课堂推送的课件, 同时雨课堂设置的思考题, 部分学生从 第 1 次得 0 分的成绩到后来基本上可以得满分的成绩。雨课堂设置思考题的成绩也在不断地提高。通 过课前推送的教学视频, 学生在课堂上能够很好地接受教学内容, 从课堂上零回答到抢着举手回答 问题, 并且还提出问题反问教师, 整个课堂师生互动感强、课堂氛围融洽; (2) 问卷网提前推送给每 位学生, 大部分学生从开始的敷衍到后来能够提出对于实验的关键问题; (3) 生物科学专业全体学 生的学习积极性和动力不断增强, 学生的平时成绩提高, 同时学生的实验操作能力也在不断地提升, 实验的成功率几乎 $100 \%$, 学生对实验学习的兴趣更加浓厚; (4) 期末课程成绩：100-90分占 $60.8 \%$, 89-80分占 35.3\%，79-70分占 3.9\%; (5) 课程结束后, 为了解 “三维” 教学模式的教学效果, 对 51 名 生物科学专业 2020 级本科生进行匿名方式调查, 回收问卷 50 份, 问卷回收率 $98.0 \%$, 结果如图4所示。 从问卷调查结果显示: (1) 雨课堂课前推送课件, $96.0 \%$ 的学生认为可以有效地帮助学生独自完成实 验, 更有 $2.0 \%$ 的学生认为非常有效地帮助独自完成实验; (2) 雨课堂设置思考题并答题, 教师批改并 考核计分, $92.0 \%$ 的学生认为有效地帮助学生对实验的理解, 更有 $2.0 \%$ 的学生认为非常有效; (3) 课 前采用问卷网收集问题, 课堂上教师针对问题及操作重点讲解, $94.0 \%$ 的学生认为可以有效地帮助学

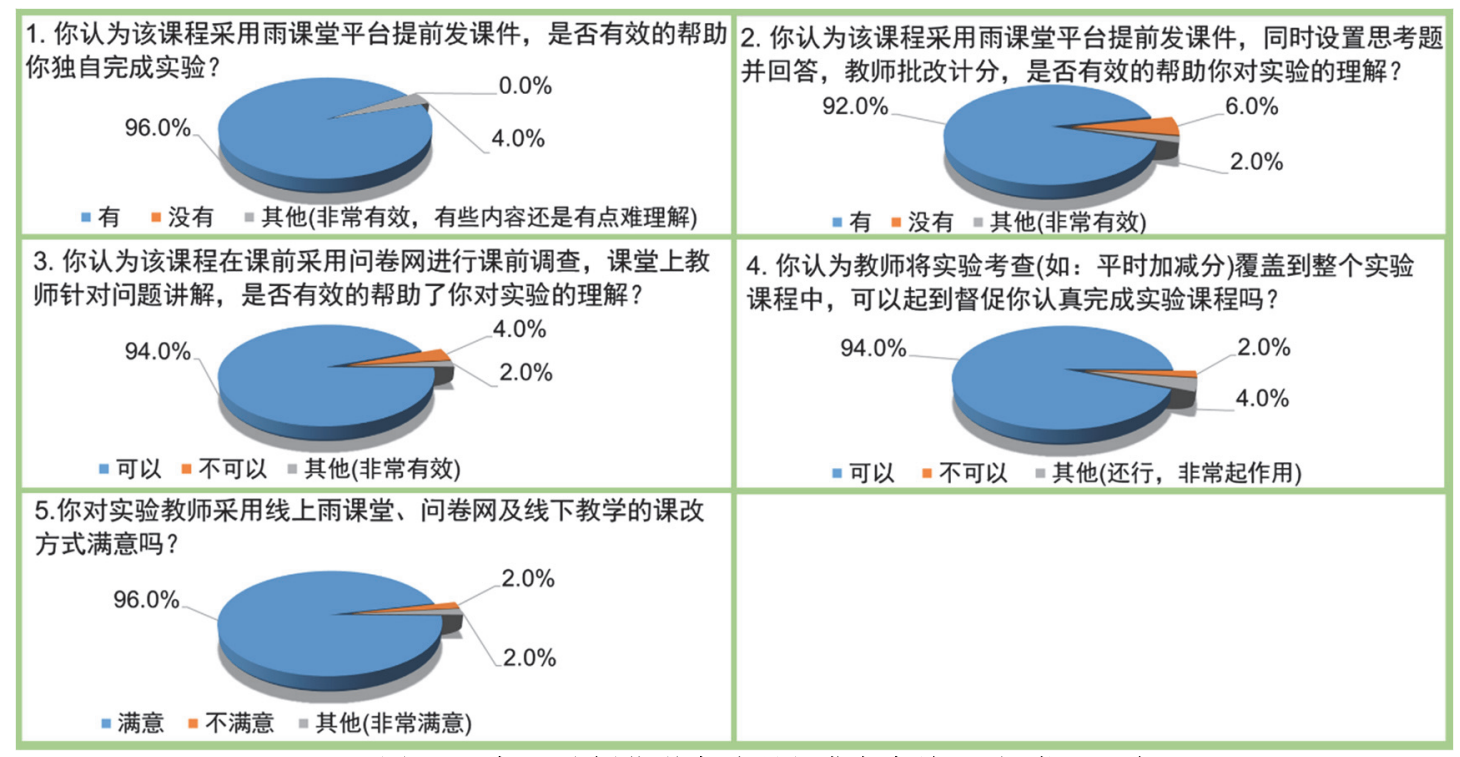

图4 无机及分析化学实验课程满意度结果-问卷网调查 
生对实验的理解, 更有 $2.0 \%$ 的学生认为非常有效; (4) 将课程考核(如: 平时加减分)覆盖到整个实验 课程中, $94.0 \%$ 的学生认为可以起到督促并认真完成实验课程的作用, 更有 $2.0 \%$ 的学生认为非常有 效; (5) 经问卷网调查显示, $98.0 \%$ 的学生满意无机及分析化学实验课程的 “三维” 教学模式。

\section{4 结语}

无机及分析化学实验 “三维” 教学模式的设计以学生为主体, 将课程考核落实到课程学习的各 个环节, 极大地提高了学生主动学习的动力和积极性, 采用加减分的方式, 提高了学生的学习动力 并激发了学生的求知欲, 同时利用雨课堂和问卷网等现代教学手段, 提高了无机及分析化学实验课 程的教学质量, 期末既考查了学生实验课程的理论知识, 又考查了学生的实际操作能力, 培养了学 生独立思考的能力、准备和实践的能力、分析和解决问题的能力, 培养了学生语言表达的能力以及 实验规范操作的能力, 使学生形成了良好的科学思维。“三维” 教学模式全面提高了课程的教学质 量, 不仅培养了学生的科研素养, 同时培养了学生团结协作意识、责任意识和开拓创新意识, 适合 在地方高校实验教学中推广。

\section{参 考 文 献}

[1] 张树永, 张剑荣, 陈六平. 大学化学, 2016, 31 (9), 1 .

[2] 董立军, 王薇, 吕东显, 兰景风. 大学化学, 2021, 36 (9), 2105056.

[3] 马雯雯, 陈庆阳, 于湛, 张红丹. 广东化工, 2020, 47 (13), 192.

[4] 于智慧, 于长顺, 宋宇, 蔡伟杰, 翟滨, 陈丽风. 广州化工, 2019, 47 (13), 180.

[5] 马海风, 刘斌, 芦昌盛, 周爱东, 田笑丛, 王风彬. 大学化学, 2019, 34 (6), 5.

[6] 梅新兰, 谢文娟, 尹智敏, 杨金风. 大学化学, 2019, 34 (9), 64 .

[7] 刘红瑜, 金谷, 姚奇志, 李娇, 李玲玲. 大学化学, 2021, 36 (2), 1912006.

[8] 郑冰, 秦川丽. 实验室研究与探索, 2020, 39 (6), 214.

[9] 宋继梅, 杨捷, 吴振玉, 吴杰颖, 田玉鹏. 实验室研究与探索, 2011, 30 (1), 106.

[10] 吴霞. 中国大学教学, 2017, No. 2, 78.

[11] 王秀云, 宿艳, 张永策, 潘玉珍. 大学化学, 2021, 36 (1), 2001032.

[12] 朱志彪, 高山. 实验室研究与探索, 2010, 29 (7), 238. 